\title{
Quantitative Structural Analysis of Nanoparticles Using Electron Pair Distribution Function (ePDF)
}

\author{
Hefei $\mathrm{Hu}^{1}$, A. M. Milinda Abeykoon ${ }^{1}$, Lijun $\mathrm{Wu}^{1}$, Yimei Zhu ${ }^{1}$ and Simon J. L. Billinge ${ }^{1,2}$ \\ 1. Condensed Matter Physics and Materials Science Department, Brookhaven National Laboratory, \\ Upton, New York 11973, USA. \\ 2. Department of Applied Physics and Applied Mathematics, Columbia University, New York, NY \\ 10027, USA.
}

Quantitatively determining the atomic arrangement of nanomaterials is essential in nanoscience. While the traditional method of X-ray crystallography fails because of limited size of coherent structural domains within nanomaterials, a specialized approach, known as the atomic pair distribution function (PDF) method, has emerged as a powerful tool to obtain quantitative 3D structural information of nanomaterials [1]. However, currently, most PDF experiments are carried out at X-ray synchrotron or neutron sources from a large user facility, where access is limited. Here, we present that electron diffraction data that are collected in a standard transmission electron microscope (TEM) can be used to quantitatively determine nanostructures by using the ePDF technique [2].

The main procedure used for quantitative structural analysis by ePDF is described as follows: the experimentally recorded 2D electron diffraction patterns are first integrated into 1D powder diffraction patterns. Then, a home-written program, PDFgetE (unpublished) is used to calculate reduced structure function, $F(Q)$, and PDF, $G(r)$. Finally, $\mathrm{G}(r)$ is modeled by using PDFgui [3] to extract structural information.

For reliable ePDF measurements, different calibration samples were tested. The NIST-standard Au nanoparticle sample is recommended to calibrate camera length (CL) and determine instrument resolution parameter, $Q_{d a m p}$, in TEM. A typical electron diffraction of the Au nanoparticle sample is shown in Fig. 1(a). Fig. 1(b) and (c) show the TEM image at low and high magnification, respectively. We can see uniform size distribution of Au nanoparticles. Once the electron diffraction of the calibration $\mathrm{Au}$ sample was recorded, our real sample, $\mathrm{SnO}_{2}$ nanoparticles, was then loaded. A typical electron diffraction of the $\mathrm{SnO}_{2}$ sample is shown in Fig. 2(a). With CL calibrated by taking the Au diffraction as a reference, the reduced structure function was calculated, which is shown in Fig. 2(b). $F(Q)$ shows good statistics up to $17 \AA^{-1}$. The resulting $\mathrm{G}(r)$ is shown as blue symbol in Fig. 2(c), and the best-fit PDF from a structural model with space group $P 4_{2} / \mathrm{mnm}$ is plotted in red with a difference curve offset below. The lattice constant $a$ and $b$ were refined to be $4.75 \AA$ and $3.23 \AA$, respectively. With the $Q_{\text {damp }}$ calibrated, the particle size was refined to be $\sim 2 \mathrm{~nm}$. Other refined parameters will also be discussed.

Furthermore, different protocols for ePDF data recording, including common/possible TEM operation mistakes, were examined by comparing the refined parameters of $\mathrm{SnO}_{2}$ with the X-ray PDF (xPDF) results, which will be discussed in the presentation.

\section{References:}

[1] T. Egami and S. J. L. Billinge, "Underneath the Bragg peaks: structural analysis of complex materials", (Pergamon Press, Elsevier). 
[2] M. Abeykoon et al, Z. Kristallogr. 227 (2012), p. 248.

[3] C. L. Farrow et al, J. Phys: condens. Mat. 19 (2007), p. 335219.

[4] Acknowledgments: This work was carried out as part of the Flucteam project at BNL supported by the US Department of Energy, Office of Science, Office of Basic Energy Sciences (DOE-BES) through account DE-AC02-98CH10886. Use of the National Synchrotron Light Source, Brookhaven National Laboratory, was supported by the DOE-BES under Contract No. DE-AC02-98CH10886.

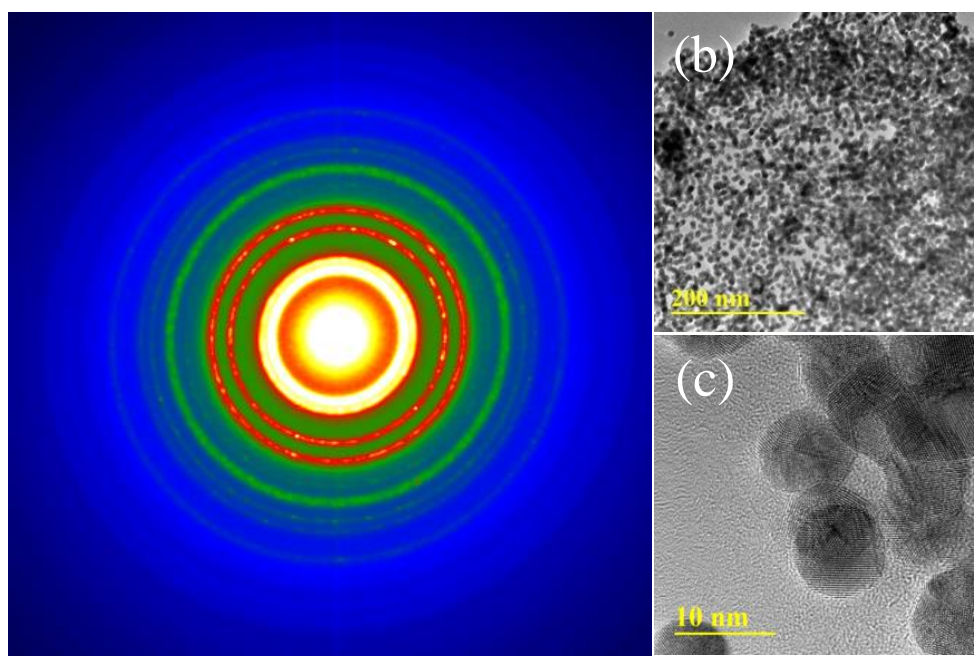

Figure 1. (a) A typical electron diffraction pattern of the $10 \mathrm{~nm}$ diameter Au nanoparticle sample. The TEM images at low and high magnification are shown in (b) and (c), respectively.
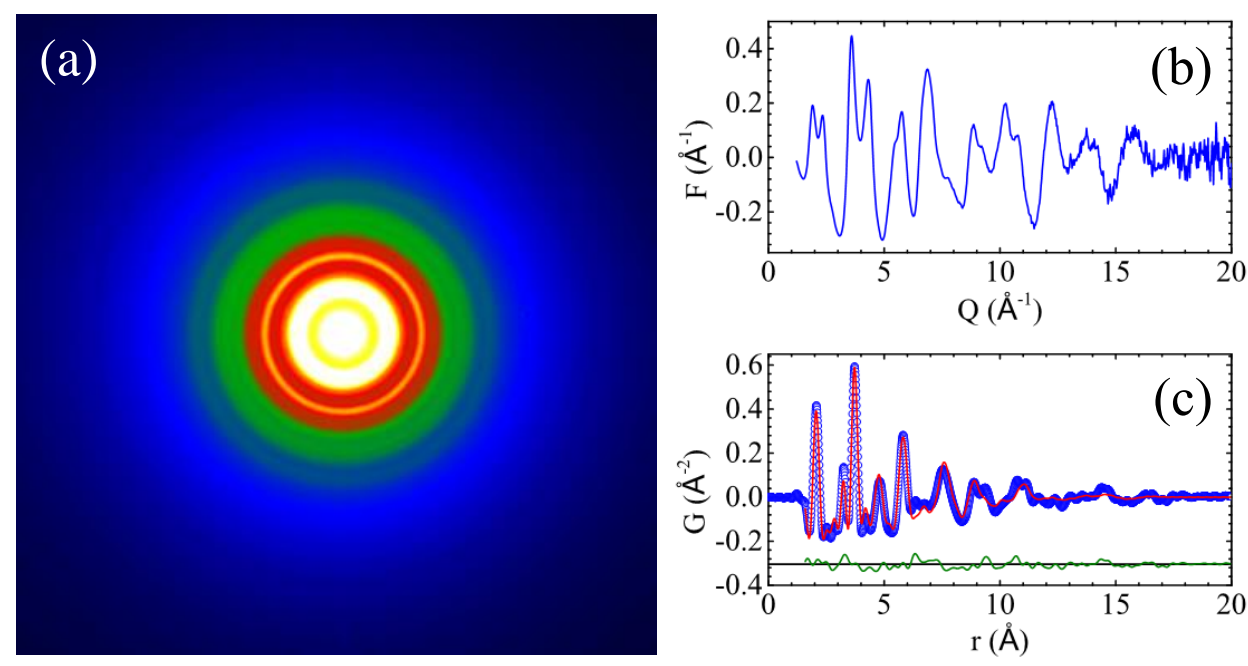

Figure 2. (a) A typical electron diffraction pattern of the $\mathrm{SnO}_{2}$ nanoparticle sample. (b) Reduced electron structure function, $F(Q)$, calculated from the integrated 2D electron diffraction pattern in (a). The resulting PDF, G(r), is shown as blue symbols in (c). The best-fit PDF from a structural model is plotted in red with a difference curve offset below. 